\title{
Generation of a novel isogenic trisomy panel in human embryonic stem cells via microcell-mediated chromosome transfer
}

\author{
Kei Hiramatsu ${ }^{\text {a }}$, Satoshi Abe ${ }^{\text {b }}$, Kanako Kazuki ${ }^{\text {b }}$, Mitsuhiko Osaki ${ }^{\text {b, c }}$, Naoyo Kajitani ${ }^{\text {b }}$, \\ Yuwna Yakura ${ }^{\text {b }}$, Mitsuo Oshimura ${ }^{\text {b }}$, Yasuhiro Kazuki a, b, ${ }^{*}$ \\ ${ }^{a}$ Department of Biomedical Science, Institute of Regenerative Medicine and Biofunction, Graduate School of Medical Science, Tottori University, 86 Nishi- \\ cho, Yonago, Tottori, 683-8503, Japan \\ ${ }^{\mathrm{b}}$ Chromosome Engineering Research Center (CERC), Tottori University, 86 Nishi-cho, Yonago, Tottori, 683-8503, Japan \\ ${ }^{\mathrm{c}}$ Division of Pathological Biochemistry, Department of Biomedical Sciences, Faculty of Medicine, Tottori University, 86 Nishi-cho, Yonago, Tottori, 683-8503, \\ Japan
}

\section{A R T I C L E I N F O}

\section{Article history:}

Received 6 November 2018

Accepted 21 November 2018

Available online 1 December 2018

\section{Keywords:}

Aneuploidy syndrome

Microcell-mediated chromosome transfer

Human embryonic stem cell

Trisomy panel

\begin{abstract}
A B S T R A C T
Aneuploidy is the gain or loss of a chromosome. Down syndrome or trisomy (Ts) 21 is the most frequent live-born aneuploidy syndrome in humans and extensively studied using model mice. However, there is no available model mouse for other congenital Ts syndromes, possibly because of the lethality of Ts in vivo, resulting in the lack of studies to identify the responsible gene(s) for aneuploid syndromes. Although induced pluripotent stem cells derived from patients are useful to analyse aneuploidy syndromes, there are concerns about differences in the genetic background for comparative studies and clonal variations. Therefore, a model cell line panel with the same genetic background has been strongly desired for sophisticated comparative analyses. In this study, we established isogenic human embryonic stem (hES) cells of Ts8, Ts13, and Ts 18 in addition to previously established Ts21 by transferring each single chromosome into parental hES cells via microcell-mediated chromosome transfer. Genes on each trisomic chromosome were globally overexpressed in each established cell line, and all Ts cell lines differentiated into all three embryonic germ layers. This cell line panel is expected to be a useful resource to elucidate molecular and epigenetic mechanisms of genetic imbalance and determine how aneuploidy is involved in various abnormal phenotypes including tumourigenesis and impaired neurogenesis.
\end{abstract}

(c) 2018 Elsevier Inc. All rights reserved.

\section{Introduction}

Aneuploidy is supernumerary gain of a chromosome (trisomy; Ts) or loss of a chromosome (monosomy). Embryonic lethality by aneuploidy accounts for approximately one-third of spontaneous miscarriages, and survivors suffer from severe diverse pathological conditions due to developmental defects [1]. Among aneuploidy syndromes, Ts13 (Patau syndrome), Ts18 (Edwards syndrome), and Ts21 (Down syndrome) are the most common congenital trisomies in humans [2]. Down syndrome (DS) is well highlighted and studied, and several DS mouse models have been generated to understand the underlying disease mechanism, but no mouse

\footnotetext{
* Corresponding author. Department of Biomedical Science, Institute of Regenerative Medicine and Biofunction, Graduate School of Medical Science, Tottori University, 86 Nishi-cho, Yonago, Tottori, 683-8503, Japan

E-mail address: kazuki@tottori-u.ac.jp (Y. Kazuki).
}

models for Ts 13 or Ts 18 have been reported $[3,4]$. Therefore, models for Ts13 and Ts18 are desired to clarify the aetiology of aneuploidy syndromes.

Induced pluripotent stem (iPS) cell technology is an extremely invaluable tool to provide models to uncover how diseases progress during development or in specific cell lineages by iPS cell differentiation [5]. Thus, several iPS cell lines have been established from patients with aneuploid syndromes and various other diseases $[1,6]$. However, it is a concern that more clonal diversity exists in iPS cell lines than in human embryonic stem (hES) cell lines, and there is difficulty to obtain an appropriate control cell line with the same genetic background $[7,8]$. Regarding Ts, although control isogenic cell lines can be obtained by spontaneous loss of a chromosome from a Ts21-derived iPS cell line [9], it is impossible to establish different Ts syndrome models with the same genetic background by iPS cell technology to investigate whether a common mechanism exists for shared phenotypic abnormalities such as mental retardation in aneuploid syndromes. Thus, studies on epigenetic 


\begin{tabular}{ll|}
\hline Abbreviations \\
Ts & trisomy \\
hES & human embryonic stem \\
DS & Down syndrome \\
iPS & induced pluripotent stem \\
AML & acute myeloid leukaemia \\
MMCT & microcell-mediated chromosome transfer \\
hChr & human chromosome; hChr.8-neo, hChr.13-neo, \\
& and hChr.18-neo, human chromosome 8, 13, and \\
& 18 tagged with a neo resistance gene \\
QH & quinacrine-Hoechst \\
FISH & fluorescence in situ hybridization \\
mFISH & multicolour FISH \\
WT & wild-type \\
SCID & severe combined immunodeficiency \\
Ts8-ES & Ts8 human embryonic stem \\
Ts13-ES & Ts13 human embryonic stem \\
Ts18-ES & Ts18 human embryonic stem \\
mES & mouse embryonic stem \\
\hline
\end{tabular}

alteration due to a trisomic state are also limited.

Aneuploidy is frequently observed in certain tumours. In particular, Ts8 is the most frequently detected Ts in acute myeloid leukaemia (AML) [10,11]. Although several lines of evidence suggest a strong causal relationship, it is unclear whether aneuploidy in tumourigenesis is the cause or result. At least, individuals with constitutional Ts8 mosaicism have an increased risk of neoplastic disorders [10]. A research approach to address these issues is chromosome transfer technology that establishes aneuploid cell lines with the same genetic background by introducing a single copy of a human chromosome into hES cells [12].

Previously, we established Ts21 cells by transferring a single copy of chromosome 21 into hES cells via microcell-mediated chromosome transfer (MMCT) [13]. This enabled us to analyse the effect of the extra copy of chromosome 21 on haematopoiesis by comparison with parental hES cells of the same genetic background. In this study, by adopting the same method, we established an isogenic cell line panel of Ts8, Ts13, and Ts18 to provide model cells for understanding the consequences of aneuploidy.

\section{Materials and methods}

Cell culture. A9 cells carrying a human chromosome 8, 13, or 18 tagged with a neo resistance gene, A9 (hChr.8-neo), (hChr.13-neo), and (hChr.18-neo) cells, respectively, were maintained in Dulbecco's modified Eagle's medium supplemented with $10 \%$ foetal bovine serum and $800 \mu \mathrm{g} / \mathrm{mL}$ G418 (Promega, Tokyo, Japan) [14-16]. A human ES (hES) line KhES-1-derived subline [13,17] was used following the human ES cell research guidelines of the Japanese government. The KhES-1-derived subline, wild-type ES (WTES), was used for MMCT. The parental WT-ES cell line and microcell hybrid clones were maintained on mitomycin C (Sigma-Aldrich, Tokyo, Japan)-treated Jcl:ICR (CLEA Japan, Tokyo, Japan) mouse embryonic fibroblasts as feeder layers in primate ES cell medium (ReproCell, Tokyo, Japan) supplemented with $4 \mathrm{ng} / \mathrm{mL}$ recombinant basic fibroblast growth factor (WAKO, Osaka, Japan).

MMCT. MMCT was performed as described previously [18]. A9 (hChr.8-neo), A9 (hChr.13-neo), and A9 (hChr.18-neo) cells were used as donor microcell hybrids. Briefly, WT-ES cells were fused with microcells prepared from donor hybrid A9 cells and selected with G418 (50 $\mu \mathrm{g} / \mathrm{mL})$. The transferred hChr.8, 13, and 18 in each line was characterised by cytogenetic analyses.

Cytogenetic analyses. Fluorescence in situ hybridization (FISH) analyses were performed in fixed metaphases of microcell hybrids using digoxigenin-labelled (Roche, Basel, Switzerland) human Cot1 DNA (Life Technologies) [18]. Chromosomal DNA was counterstained with DAPI (Sigma-Aldrich). Images were captured using an AxioImagerZ2 fluorescence microscope (Carl Zeiss GmbH, Jena, Germany). Slides of microcell hybrids were stained with quinacrine mustard and Hoechst 33258 to enumerate chromosomes. Images were captured under the AxioImagerZ2 fluorescence microscope and analysed with the Ikaros software program (MetaSystems, Altlussheim, Germany). Multicolour FISH (mFISH) analyses were performed in accordance with the manufacturer's instructions (MetaSystems). Human mFISH probes were purchased from MetaSystems $\mathrm{GmbH}$. Metaphase images were captured digitally with a CoolCubeI CCD camera and the ISIS mFISH software program (MetaSystems).

Microarray analyses. Total RNA from parental human embryonic stem cells (WT-ES), and Ts8, 13, and 18 human embryonic stem cell lines (Ts8-ES, Ts13-ES, and Ts18-ES, respectively) was prepared using RNeasy (Qiagen, Hilden, Germany), according to the manufacturer's instructions. Microarray analyses were performed using a 3D-Gene Human Oligo chip 25k (Toray Industries Inc., Tokyo, Japan). Microarray slides were scanned using a 3D-Gene Scanner (Toray Industries) and processed by 3D-Gene Extraction software (Toray Industries).

Teratoma formation and histology. To produce teratomas, $1 \times 10^{6}$ WT-ES, Ts8-ES, Ts13-ES and Ts18-ES cells were subcutaneously injected into testes of severe combined immunodeficiency (SCID) mice (Charles River, Yokohama, Japan). After 8 weeks, resected teratomas were fixed in $20 \%$ formalin, processed for paraffin sectioning, and then stained with haematoxylin and eosin. All animal experiments were approved by the Institutional Animal Care and Use Committee of Tottori University.

\section{Results}

We established Ts8, Ts13, and Ts18 human ES (hES) cell lines as a cell line panel with the same genetic background by transferring a single copy of a human chromosome into hES cells via MMCT (Fig. 1). We recently established an improved chromosome transfer technology applicable to transfer of a chromosome into hES cells [13]. As donor cell lines, we used a library of human/mouse A9 monochromosomal hybrids in which a single human chromosome tagged with a neo resistance gene for MMCT was maintained independently [14-16]. First, we performed fluorescence in situ hybridization (FISH) analyses to confirm whether human chromosome 8 (hChr.8), 13 (hChr.13), and 18 (hChr.18) were stably and independently maintained in each mouse A9 cell line. Regarding the A9 clone carrying a hChr.8 tagged with a neo resistance gene (hChr.8-neo), FISH analyses revealed that the A9 cells contained two independent hChr.8 and one hChr.8 fragment translocated to the host genome (Fig. 2A). Furthermore, FISH analyses showed that the A9 clone carrying a hChr.13 tagged with the neo resistance gene (hChr.13-neo) and A9 clone carrying a hChr.18 tagged with the neo resistance gene (hChr.18-neo) contained a hChr.13 and hChr.18, respectively, which were independently maintained in host cells (Fig. 2A).

Next, we performed MMCT into wild-type hES (WT-ES) cells using A9 (hChr.8-neo), A9 (hChr.13-neo), and A9 (hChr.18-neo) cells as donors. We performed 16, four, and two fusions with A9 (hChr.8neo), A9 (hChr.13-neo), and A9 (hChr.18-neo) cells, respectively. After 17-20 days under G418 selection, 20, two, and five drugresistant hES cell clones were obtained for Ts8, Ts13, and Ts18, respectively (Ts8-ES, Ts13-ES, and Ts18-ES, respectively). Their 


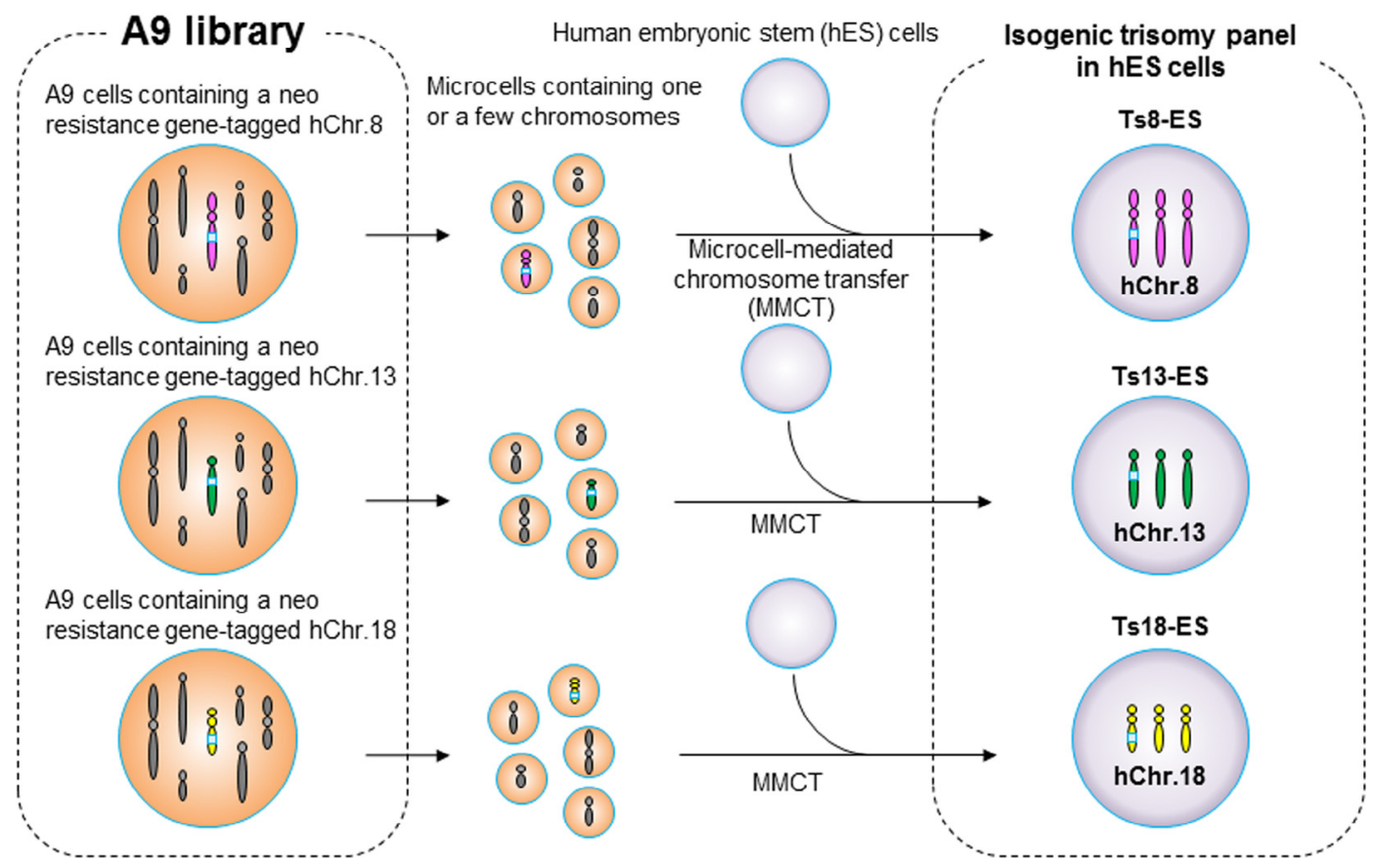

Fig. 1. Generation of an isogenic trisomy panel in hES cells by microcell-mediated chromosome transfer. Schematic diagram of the establishment of Ts8, Ts13, and Ts18 in hES cells.

A

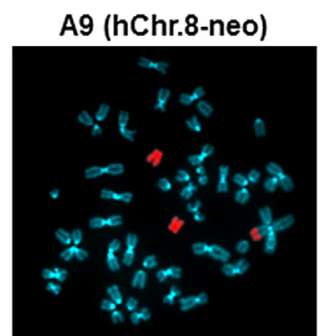

A9 (hChr.13-neo)

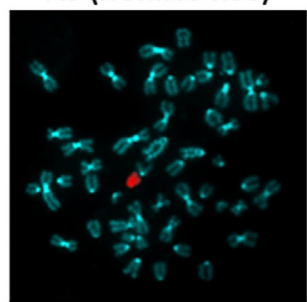

A9 (hChr.18-neo)

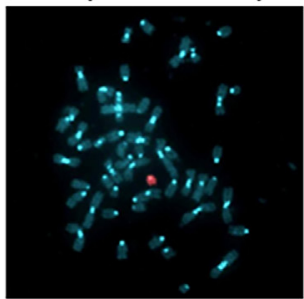

B WT-ES

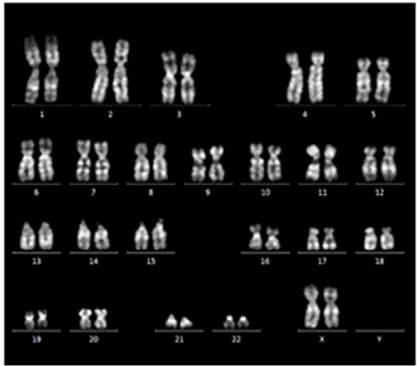

C 8888

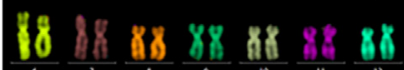
ad on $\frac{10}{4}$ $\frac{8 X}{48} \frac{86}{10}$ III 88 . 18 . 88
Ts8-ES

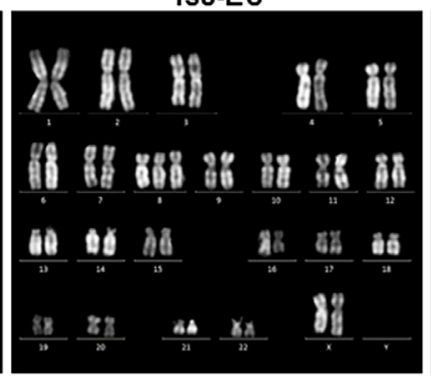

Ts8-ES

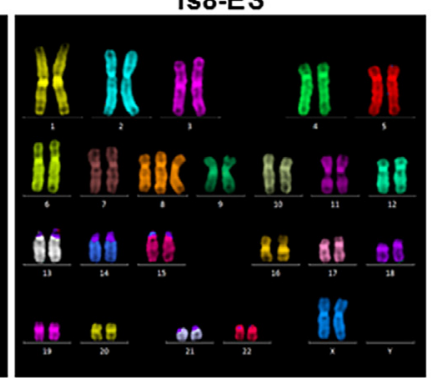

Ts13-ES

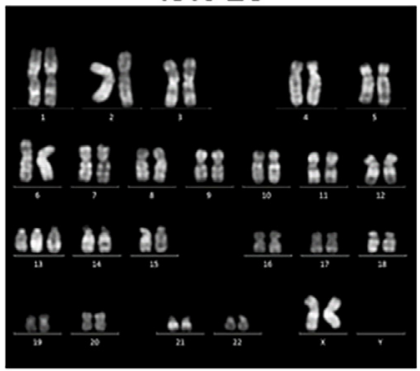

Ts13-ES

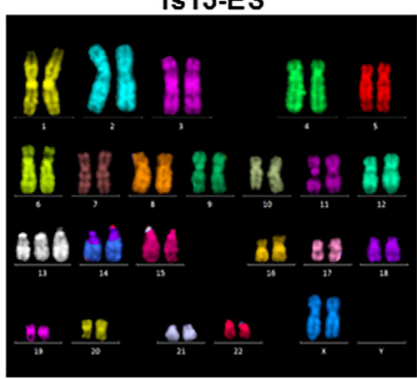

Ts18-ES

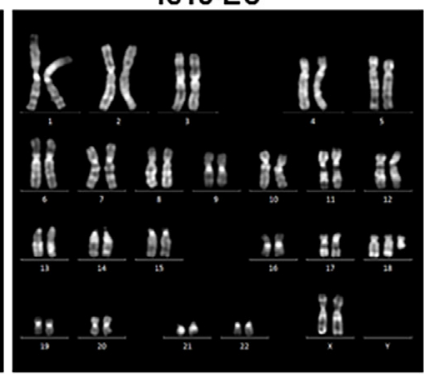

Ts18-ES

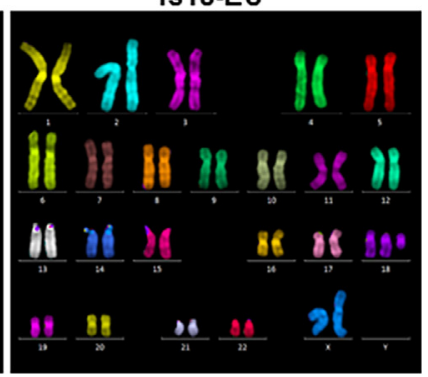

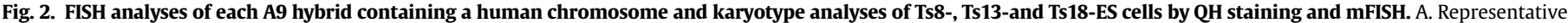

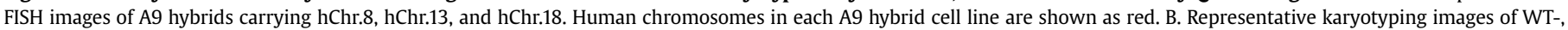
Ts8-, Ts13-, Ts18-ES cell lines by QH staining. C. Representative karyotyping images of WT-, Ts8-, Ts13-, Ts18-ES cell lines by mFISH analyses. 

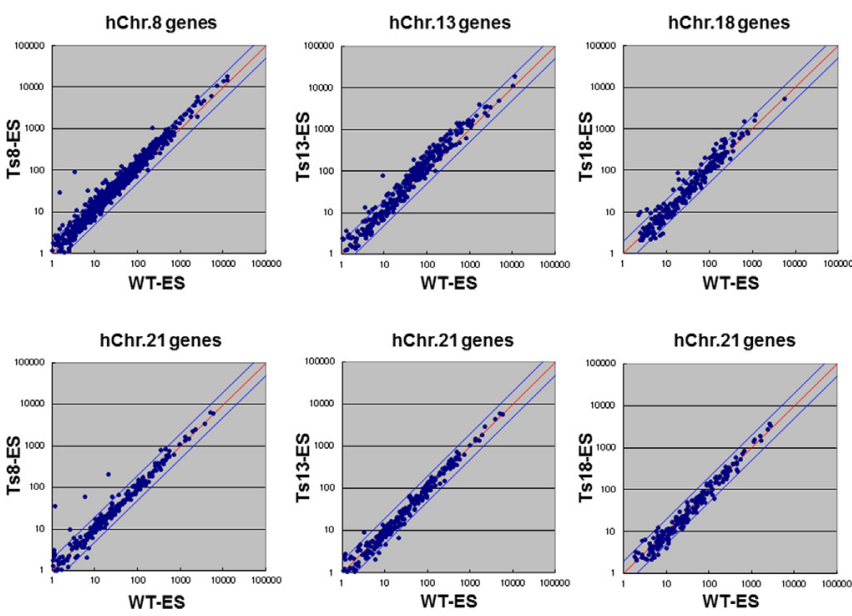

Fig. 3. Gene expression analyses of Ts8-, Ts13-, and Ts18-ES cells. Microarray analyses of WT-, Ts8-, Ts13-, and Ts18-ES cells. Representative comparison data of genes on hChr.8, 13, and 18 (upper panel) and hChr.21 (lower panel) between WT-ES and each Ts-ES cell line are shown. The dots between blue and red lines, and the dots on red line show genes within 2-fold differences in expression and equal expression, respectively.

karyotypes were analysed by quinacrine-Hoechst $(\mathrm{QH})$ staining. In Ts8-, Ts13-and Ts18-ES clones, QH staining indicated that the karyotypes were $47, \mathrm{XX},+8,47, \mathrm{XX},+13$, and $47, \mathrm{XX},+18 \mathrm{q}$ - in four, two, and five clones out of 12 , two, and five analysed clones, respectively (Fig. 2B). Ts18-ES clones had partial trisomy in which the q arm of the transferred human chromosome 18 showed partial deletion. Regarding Ts8-and Ts13-ES clones, the transferred chromosomes appeared to be intact. Multicolour fluorescence in situ hybridization analyses (mFISH) were performed in Ts8-, Ts13-and Ts18-ES clones to confirm successful transfer of a single exogenous human chromosome into WT-ES cells. There was apparently no host chromosome abnormalities in the selected Ts8-, Ts13-and Ts18-ES cell clones (Fig. 2C). These results suggested that each single chromosome was successfully transferred into WT-ES cells.

Microarray analyses revealed that genes on hChr.8, hChr.13, and hChr.18 in Ts8-ES, Ts13-ES and Ts18-ES cells, respectively, were globally overexpressed, but gene expression from hChr.21 was comparable with that in WT-ES cells (Fig. 3). These data suggested that each exogenous human chromosome was successfully transferred into WT-ES cells.

To determine whether Ts8-ES, Ts13-ES, and Ts18-ES cells could differentiate into all three embryonic germ layers, two clones of each Ts8-ES, Ts13-ES and Ts18-ES cell line were injected into testes of severe combined immunodeficiency (SCID) mice. Histological
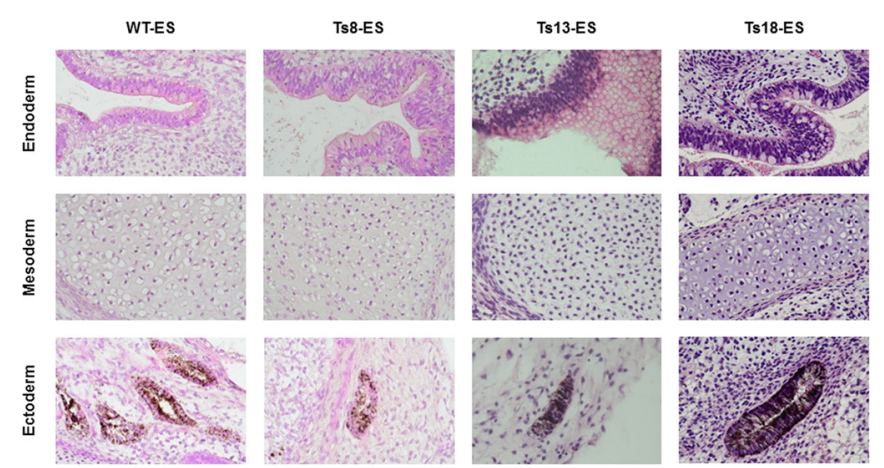

Fig. 4. Teratoma formation assays of Ts8-, Ts13-, and Ts18-ES cells. Teratomas derived from WT-, Ts8-, Ts13-, and Ts18-ES cells. Eight weeks after cell transplantation, structures originating from all three germ layers were found in the teratomas. analyses revealed all three embryonic germ layers (endoderm, mesoderm, and ectoderm) in all teratomas derived from all Ts8-ES, Ts13-ES, and Ts18-ES cell clones (Fig. 4). Taken together, these results suggested that the obtained Ts8-ES, Ts13-ES, and Ts18-ES cell clones were pluripotent.

\section{Discussion}

In this study, we successfully transferred a single human chromosome into hES cells and established a trisomy hES cell line panel including Ts8, Ts13, and Ts18 with the same genetic background. Cytogenetic analyses confirmed that an additional hChr.8, hChr.13, and hChr.18 were stably and independently maintained in hES cells, and the host chromosome was not disrupted during the process of MMCT. Microarray analyses further confirmed that the genes at least in the trisomic regions were overexpressed, and teratoma formation assays confirmed that each trisomy cell line had a differentiation potential. This cell line panel with the same genetic background enables comparison of the effect of each trisomy on several diseases more accurately using recent advanced pluripotent stem cell differentiation technologies that have given rise to several cell types. Although iPS cell technology is a powerful tool to produce disease-specific pluripotent stem cells including aneuploidy, there is a concern that the obtained clones have variation in their epigenetic status, which affects differentiation fate [19].

Regarding chromosome transfer, although mouse A9 cells are useful donor cells for MMCT because of a high level of microcell formation capacity, the transfer efficiency of a single chromosome into hES cells is considerably low. It is possible to increase the efficiency using $\mathrm{CHO}$ cells as donors in combination with advanced virus envelope-based fusion technologies reported recently [20-22].

Mental retardation is the common phenotype of congenital aneuploidy patients $[23,24]$. We previously reported that mouse embryonic stem (mES) cells with autosomal aneuploidy (Ts1-mES and Ts8-mES) show augmented apoptosis during early neuronal differentiation induced by SDIA treatment [25]. Commonly downregulated genes were identified and confirmed by microarray and real-time qRT-PCR. Furthermore, downregulation of targeted genes in WT-mES cells by siRNA recapitulated the augmented apoptosis during early neurogenesis as seen in mES cells with autosomal aneuploidy. These results suggested that the autosomal imbalance associated with general neuronal loss was due to a common apoptosis mechanism. Although NPCs derived from monozygotic twins discordant for Ts21 also showed increased apoptosis, whether the phenomena and mechanism observed in mES cells is common in humans remains to be elucidated [26]. This may be addressed using WT-, Ts8-, Ts13-, and Ts18-ES cells in addition to Ts21-ES cells that we established previously [13].

It is still controversial whether aneuploidy is the cause or consequence of tumourigenesis. Ts8 as a sole chromosome aberration is frequently observed in acute myeloid leukaemia (AML) and accounts for approximately 5\% of cytogenetically abnormal cases [10]. However, much remains to be elucidated regarding its association with leukaemogenesis. A comparative study of WT-ES and Ts8-ES cells by differentiation to hematopoietic cells is expected to provide striking information on how Ts8 contributes to leukaemogenesis. Recently, genome editing studies validating the contribution of recurrent mutations to colorectal carcinogenesis suggested that aneuploidy, as the gain of chromosomes such as 7 , 13 , and 20 , may be associated with aggressive tumour phenotypes $[27,28]$. Thus, genome editing technologies applied to Ts8-ES cells may be able to confirm critical genetic aberration of Ts8-associated leukaemogenesis. In addition, Ts8 is common together with other chromosomal abnormalities in many types of neoplastic disorders 
[10]. Therefore, the Ts8-ES cell line is useful to uncover the mechanism of tumourigenesis associated with multiple chromosomal aberrations by transfer of an additional chromosome to the Ts8-ES cell line via MMCT.

Identification of the gene responsible for specific lesions by analyses of WT-, Ts8-, Ts13-, Ts18-and Ts21-ES cells may provide critical information to cure aneuploid syndromes. Because genome editing technologies enable modification of chromosomes with high efficiency, candidate genes and genomic regions responsible for trisomy syndromes based on comprehensive analysis data can be confirmed by disruption or deletion of such genes or regions on the transferred chromosomes via genome editing [29]. To avoid offtarget effects on the host genome, there is a method to transfer a modified chromosome after disruption or deletion in A9 cells by genome editing, because off-target effects on the host genome in A9 cells as donor cells are almost negligible.

Taken together, the cell line panel with the same genetic background established in this study will provide information to elucidate the mechanism of aneuploid syndromes including impaired neurogenesis and tumourigenesis. The isogenic trisomy cell panel established by chromosome transfer technology is an invaluable tool for biomedical research such as studies on dosage effects of genetic materials.

\section{Funding}

This study was supported in part by JSPS KAKENHI Grant Number 25221308 (M.O.) and the Regional Innovation Support Program from the Ministry of Education, Culture, Sports, Science and Technology of Japan (M.O. and Y.K.).

\section{Competing financial interests}

The authors declare no competing financial interest.

\section{Acknowledgements}

We thank T. Yoshino, H. Kohno, Y. Sumida, E. Kaneda, and C. Ishihara at Tottori University for technical assistance and Drs. $\mathrm{H}$. Kugoh, T. Ohira, N. Uno, M. Hiratsuka, and H. Satofuka at Tottori University for critical discussions. We also thank Mitchell Arico from Edanz Group (www.edanzediting.com/ac) for editing a draft of this manuscript. This research was partly performed at the Tottori Bio Frontier managed by Tottori prefecture.

\section{References}

[1] W. Li, X. Wang, W. Fan, P. Zhao, Y.C. Chan, S. Chen, S. Zhang, X. Guo, Y. Zhang, Y. Li, J. Cai, D. Qin, X. Li, J. Yang, T. Peng, D. Zychlinski, D. Hoffmann, R. Zhang, K. Deng, K.M. Ng, B. Menten, M. Zhong, J. Wu, Z. Li, Y. Chen, A. Schambach, H.F. Tse, D. Pei, M.A. Esteban, Modeling abnormal early development with induced pluripotent stem cells from aneuploid syndromes, Hum. Mol. Genet. 21 (2012) 32-45.

[2] B. Liu, S. Filippi, A. Roy, I. Roberts, Stem and progenitor cell dysfunction in human trisomies, EMBO Rep. 16 (2015) 44-62.

[3] M. Gupta, A.R. Dhanasekaran, K.J. Gardiner, Mouse models of Down syndrome: gene content and consequences, Mamm. Genome 27 (2016) 538-555.

[4] O. Sheppard, F.K. Wiseman, A. Ruparelia, V.L. Tybulewicz, E.M. Fisher, Mouse models of aneuploidy, ScientificWorldJournal 2012 (2012) 214078.

[5] K. Takahashi, K. Tanabe, M. Ohnuki, M. Narita, T. Ichisaka, K. Tomoda, S. Yamanaka, Induction of pluripotent stem cells from adult human fibroblasts by defined factors, Cell 131 (2007) 861-872.

[6] I.H. Park, N. Arora, H. Huo, N. Maherali, T. Ahfeldt, A. Shimamura, M.W. Lensch, C. Cowan, K. Hochedlinger, G.Q. Daley, Disease-specific induced pluripotent stem cells, Cell 134 (2008) 877-886.

[7] S. Yokobayashi, K. Okita, M. Nakagawa, T. Nakamura, Y. Yabuta, T. Yamamoto, M. Saitou, Clonal variation of human induced pluripotent stem cells for induction into the germ cell fate, Biol. Reprod. 96 (2017) 1154-1166.

[8] M. Ohnuki, K. Takahashi, Present and future challenges of induced pluripotent stem cells, Philos. Trans. R. Soc. Lond. B Biol. Sci. 370 (2015) 20140367.
[9] G.A. Maclean, T.F. Menne, G. Guo, D.J. Sanchez, I.H. Park, G.Q. Daley, S.H. Orkin, Altered hematopoiesis in trisomy 21 as revealed through in vitro differentiation of isogenic human pluripotent cells, Proc. Natl. Acad. Sci. U. S. A. 109 (2012) 17567-17572.

[10] K. Paulsson, B. Johansson, Trisomy 8 as the sole chromosomal aberration in acute myeloid leukemia and myelodysplastic syndromes, Pathol. Biol. 55 (2007) 37-48.

[11] C. Schoch, A. Kohlmann, M. Dugas, W. Kern, S. Schnittger, T. Haferlach, Impact of trisomy 8 on expression of genes located on chromosome 8 in different AML subgroups, Genes Chromosomes Cancer 45 (2006) 1164-1168.

[12] M. Oshimura, N. Uno, Y. Kazuki, M. Katoh, T. Inoue, A pathway from chromosome transfer to engineering resulting in human and mouse artificial chromosomes for a variety of applications to bio-medical challenges, Chromosome Res. 23 (2015) 111-133.

[13] Y. Kazuki, Y. Yakura, S. Abe, M. Osaki, N. Kajitani, K. Kazuki, S. Takehara, K. Honma, H. Suemori, S. Yamazaki, T. Sakuma, T. Toki, R. Shimizu, H. Nakauchi, T. Yamamoto, M. Oshimura, Down syndrome-associated haematopoiesis abnormalities created by chromosome transfer and genome editing technologies, Sci. Rep. 4 (2014) 6136.

[14] J. Inoue, K. Mitsuya, S. Maegawa, H. Kugoh, M. Kadota, D. Okamura, T. Shinohara, S. Nishihara, S. Takehara, K. Yamauchi, T.C. Schulz, M. Oshimura, Construction of 700 human/mouse A9 monochromosomal hybrids and analysis of imprinted genes on human chromosome 6, J. Hum. Genet. 46 (2001) 137-145.

[15] H. Kugoh, K. Mitsuya, M. Meguro, K. Shigenami, T.C. Schulz, M. Oshimura, Mouse A9 cells containing single human chromosomes for analysis of genomic imprinting, DNA Res. 6 (1999) 165-172.

[16] N. Uno, K. Hiramatsu, K. Uno, S. Komoto, Y. Kazuki, M. Oshimura, CRISPR/Cas9induced transgene insertion and telomere-associated truncation of a single human chromosome for chromosome engineering in CHO and A9 cells, Sci. Rep. 7 (2017) 12739.

[17] K. Hasegawa, T. Fujioka, Y. Nakamura, N. Nakatsuji, H. Suemori, A method for the selection of human embryonic stem cell sublines with high replating efficiency after single-cell dissociation, Stem Cell. 24 (2006) 2649-2660.

[18] T. Shinohara, K. Tomizuka, S. Miyabara, S. Takehara, Y. Kazuki, J. Inoue, M. Katoh, H. Nakane, A. Iino, A. Ohguma, S. Ikegami, K. Inokuchi, I. Ishida, R.H. Reeves, M. Oshimura, Mice containing a human chromosome 21 model behavioral impairment and cardiac anomalies of Down's syndrome, Hum. Mol. Genet. 10 (2001) 1163-1175.

[19] M. Nishizawa, K. Chonabayashi, M. Nomura, A. Tanaka, M. Nakamura, A. Inagaki, M. Nishikawa, I. Takei, A. Oishi, K. Tanabe, M. Ohnuki, H. Yokota, M. Koyanagi-Aoi, K. Okita, A. Watanabe, A. Takaori-Kondo, S. Yamanaka, Y. Yoshida, Epigenetic variation between human induced pluripotent stem cell lines is an indicator of differentiation capacity, Cell Stem Cell 19 (2016) $341-354$.

[20] M. Hiratsuka, K. Ueda, N. Uno, K. Uno, S. Fukuhara, H. Kurosaki, S. Takehara, M. Osaki, Y. Kazuki, Y. Kurosawa, T. Nakamura, M. Katoh, M. Oshimura, Retargeting of microcell fusion towards recipient cell-oriented transfer of human artificial chromosome, BMC Biotechnol. 15 (2015) 58.

[21] M. Katoh, Y. Kazuki, K. Kazuki, N. Kajitani, M. Takiguchi, Y. Nakayama, T. Nakamura, M. Oshimura, Exploitation of the interaction of measles virus fusogenic envelope proteins with the surface receptor CD46 on human cells for microcell-mediated chromosome transfer, BMC Biotechnol. 10 (2010) 37.

[22] T. Suzuki, Y. Kazuki, M. Oshimura, and T. Hara, Highly efficient transfer of chromosomes to a broad range of target cells using Chinese hamster ovary cells expressing murine leukemia virus-derived envelope proteins, PloS One 11 (2016) e0157187.

[23] C.J. Epstein, The Consequences of Chromosome Imbalance: Principles, Mechanisms, and Models, Cambridge University Press, New York, NY, 1986.

[24] M. Kadota, R. Nishigaki, C.C. Wang, T. Toda, Y. Shirayoshi, T. Inoue, T. Gojobori, K. Ikeo, M.S. Rogers, M. Oshimura, Proteomic signatures and aberrations of mouse embryonic stem cells containing a single human chromosome 21 in neuronal differentiation: an in vitro model of Down syndrome, Neuroscience 129 (2004) 325-335.

[25] Y. Kai, C.C. Wang, S. Kishigami, Y. Kazuki, S. Abe, M. Takiguchi, Y. Shirayoshi, T. Inoue, H. Ito, T. Wakayama, M. Oshimura, Enhanced apoptosis during early neuronal differentiation in mouse ES cells with autosomal imbalance, Cell Res. 19 (2009) 247-258.

[26] Y. Hibaoui, I. Grad, A. Letourneau, M.R. Sailani, S. Dahoun, F. A Santoni, S. Gimelli, M. Guipponi, M.F. Pelte, F. Bena, S.E. Antonarakis, A. Feki, Modelling and rescuing neurodevelopmental defect of Down syndrome using induced pluripotent stem cells from monozygotic twins discordant for trisomy 21, EMBO Mol. Med. 6 (2014) 259-277.

[27] M. Matano, S. Date, M. Shimokawa, A. Takano, M. Fujii, Y. Ohta, T. Watanabe, T. Kanai, T. Sato, Modeling colorectal cancer using CRISPR-Cas9-mediated engineering of human intestinal organoids, Nat. Med. 21 (2015) 256-262.

[28] J. Drost, R.H. van Jaarsveld, B. Ponsioen, C. Zimberlin, R. van Boxtel, A. Buijs, N. Sachs, R.M. Overmeer, G.J. Offerhaus, H. Begthel, J. Korving, M. van de Wetering, G. Schwank, M. Logtenberg, E. Cuppen, H.J. Snippert, J.P. Medema, G.J. Kops, H. Clevers, Sequential cancer mutations in cultured human intestinal stem cells, Nature 521 (2015) 43-47.

[29] N. Uno, S. Abe, M. Oshimura, Y. Kazuki, Combinations of chromosome transfer and genome editing for the development of cell/animal models of human disease and humanized animal models, J. Hum. Genet. 63 (2018) 145-156. 